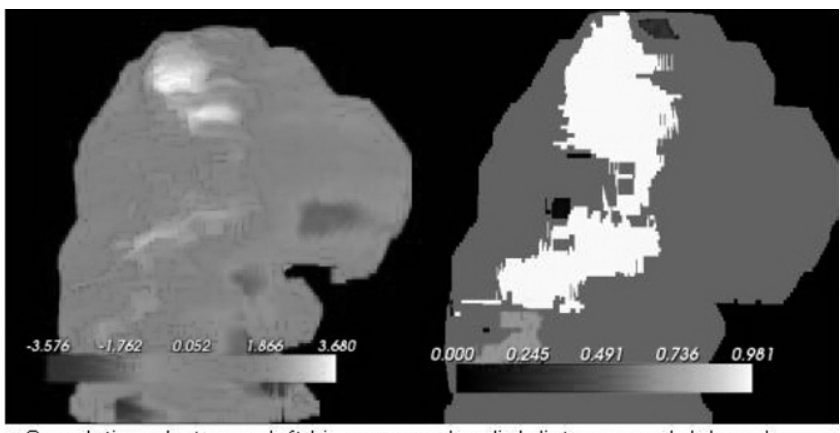

Correlations between left hippocampal radial distance and delayed memory peformance. Left panel uncorrected t-values; right panel cluster analysis. White denotes area of corrected $p$ value $=0.019$.

IC-P2-098 FREQUENCY OF HEMISPHERIC METABOLIC ASYMMETRY IN PROBABLE ALZHEIMER'S DISEASE

Norman L. Foster ${ }^{1}$, Angela Y. Wang ${ }^{1}$, James A. Levy ${ }^{1}$,

Robert A. Koeppe ${ }^{2}$, William J. Jagust ${ }^{3}$, Kewei Chen ${ }^{4}$, Eric M. Reiman ${ }^{4}$, Michael W. Weiner ${ }^{5},{ }^{1}$ University of Utah, Salt Lake City, UT, USA;

${ }^{2}$ University of Michigan, Ann Arbor, MI, USA; ${ }^{3}$ University of California, Berkeley, Berkeley, CA, USA; ${ }^{4}$ Banner Alzheimer's Institute, Phoenix,

AZ, USA; ${ }^{5}$ University of California, San Francisco, San Francisco, CA, USA. Contact e-mail: norman.foster@hsc.utah.edu

Background: Alzheimer's disease (AD) causes, on average, a symmetric pattern of glucose hypometabolism. However, some patients have hypometabolism predominantly affecting either the left or right hemisphere. These patients may have atypical presentations that could cause them to be systematically excluded from clinical drug trials. It is unknown whether the left or right hemisphere is more likely to be selectively affected. Furthermore, the frequency of significant hemispheric metabolic asymmetry is unknown. Methods: We evaluated 95 baseline FDG-PET scans in patients with clinically diagnosed mild AD (MMSE 20-27) enrolled in the Alzheimer's Disease Neuroimaging Initiative (ADNI). Each scan was analyzed with Neurostat, providing 3D-stereotactic surface projection (3D-SSP) maps of glucose metabolism relative to pons and corresponding statistical maps of Z-scores computed in comparison to 27 cognitively normal elderly control subjects. We visually evaluated the pattern of glucose hypometabolism, considering 5 regions in each hemisphere typically affected in dementia. Hemispheric asymmetry was considered significant when hypometabolism was 2 or more standard deviations greater in one hemisphere. We also calculated 3D-SSP maps of mean rates of glucose metabolism and Z-score maps. Results: Ten patients had an FTD-like pattern of hypometabolism and were excluded from further analyses. In the remaining $85 \mathrm{AD}$ subjects, FDG-PET images were symmetric in 70 $(82.4 \%)$. Hypometabolism predominantly involved left hemisphere regions in $8(9.4 \%)$ and predominantly involved right hemisphere regions in $7(8.2 \%)$. These groups did not differ significantly in age or dementia severity as measured by MMSE. Impairment was greater in naming and word fluency for patients with predominant left hemisphere hypometabolism and clock drawing scores were lowest for those with predominant right hemisphere hypometabolism. Conclusions: Although AD patients as a group have completely symmetric glucose hypometabolism, individual patterns vary reflecting cognitive differences. In this sample, roughly similar proportions of left and right predominant hypometabolism was seen in a minority of patients. The frequency of metabolic asymmetry may differ in community samples. The mechanisms accounting for selective involvement of one hemisphere are unexplained and need to be explored further. Supported in part by the Center for Alzheimer's Care, Imaging and Research and NIH grant AG024904.

\section{IC-P2-099 SYSTEMATICS OF THE ADNI PHANTOM}

Jeff L. Gunter ${ }^{1}$, Bret Borowski ${ }^{1}$, Matt Bernstein ${ }^{1}$, Chadwick Ward ${ }^{1}$, Paula Britson ${ }^{1}$, Joel Felmlee ${ }^{1}$, Norbert Schuff ${ }^{2}$, Michael Weiner ${ }^{2}$, Clifford R. Jack ${ }^{1}$, The Alzheimer's Disease Neuroimaging Initiative (ADNI), ${ }^{1}$ Mayo Clinic and Foundation, Rochester, MN, USA; ${ }^{2}$ UC San Francisco and Magnetic Resonance Unit (114M), San Francisco, CA, USA. Contact e-mail: gunter.jeffrey@mayo.edu

Background: The Alzheimer's Disease Neuroimaging Initiative (ADNI) employs a fleet of 66 phantoms (photo below) for MRI scanner characterization and to insure the integrity of data pre-processing steps. Eighty-four different scanners are used in ADNI. Methods: The ADNI phantom is scanned along with the subject for every ADNI MRI exam. Geometric fidelity is assessed by comparing the design with observed locations of 160 small spherical fiducial markers. Differences in expected and observed positions are plotted versus observed positions below. SNR and relative contrast are estimated from larger spheres containing solutions of known copper sulfate concentration. Phantom images are acquired with the same MP-RAGE pulse sequence used for human imaging. The phantom scan analysis is fully automated and provides estimates of gradient calibration (proportional to an object's size as measured by MRI), non-linear geometric distortion, SNR and contrast properties. Estimates of within phantom measurement stability were obtained by repeated scanning. Estimates of phantom to phantom variability were obtained by comparing all phantoms to single phantom. Results: Repeatability was assessed by scanning a single phantom multiple times; between scans the phantom was removed and replaced. Coefficients of variation (CV) for SNR and contrast measures were found in the 2-4\% range. Gradient calibration CV's were in the range of $3 \times 10^{-4}$ to $5 \times 10^{-4}$, the larger $\mathrm{CV}$ in the scan frequency encoding direction. The phantom construction is a stacked plate design. Phantom to phantom variation is smallest parallel to the plates. Observed CV's parallel to the plate are essentially the same as the within phantom measurement error (i.e. withinplate the manufacturing variability is likely smaller than the measurement error). The $\mathrm{CV}$ perpendicular to the plate is $15 \times 10^{-4}$ ( $3 \mathrm{X}$ to $5 \mathrm{X}$ larger than measurement error), and is assumed to be due to variation in the plate stacking during manufacturing. Conclusions: The leading source of potential error in gradient measurements using the ADNI phantom is phantom-to-phantom variability. Owing to the phantom design, that variability is anisotropic. Given a multi-year study, with thousands of MRI studies, phantoms will occasionally be damaged (dropped) and must be replaced introducing a confound in the consistency of calibration measurements.

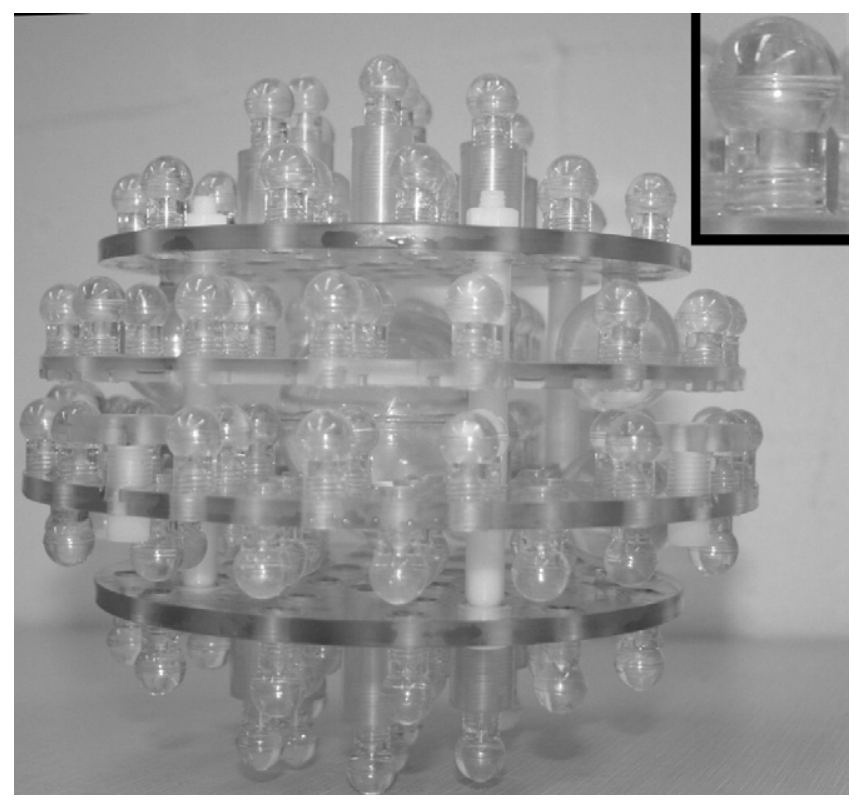

